\title{
Quo vadis Geographiedidaktik (II): Was die Fishbowl-Diskussion auf dem HGD-Symposium in Jena (2017) an Perspektiven und Grenzen aufzeigte
}

(mił Beiträgen von Ute Wardenga, Leif Mönter, Uwe Schulze, Marion Plien, Eva Nöthen, Verena Schreiber, Thomas Jekel, Ingrid Hemmer, Stephan Schurig, Lukas Recknagel, Detlef Kanwischer und Johanna Benz) ${ }^{2}$

"itta.bauer@geo.uzh.ch, Institut für Geographie, Universität Zürich
"*inga.gryl@uni-due.de, Institut für Geographie \& Institut für Sachunterricht, Universität Duisburg-Essen

eingereicht am: 27.02.2018, akzeptiert am: 14.07.2018

Der Beitrag fasst in konzentrierter Weise den Verlauf und die Ergebnisse der Fishbowl-Diskussion auf dem HGD-Symposium in Jena (2017) zur Frage nach den Perspektiven und Grenzen geographiedidaktischer Forschung zusammen. Die Besonderheit des Textes liegt in seiner methodischen Anlage: Bei der Verschriftlichtung der Diskussionsbeiträge konnten sich die Teilnehmer/innen an einer Form eines kooperativen Schreibprozesses beteiligen und so den inhaltlichen Diskurs fortführen und konkretisieren.

Keywords: Geographiedidaktik, Entwicklungslinien, Fachdiskurs, HGD-Symposium

\section{Opportunities and limitations in geography didactics: conclusions drawn from the fishbowl conversation at the 2017 HGD-symposium in Jena}

The article poigantly summarizes the main discourses and the results of a fishbowl conversation held at HGD-symposium in Jena (2017), focusing on current perspectives and future challenges of research in geography didactics. Hereby, an innovative format is put to the test: a form of cooperative writing. The participants of the discussion were offered to take part in editing their oral contributions and commenting on a draft version of the full text aimed at intensifying the debate about geography and geography didactics.

Keywords: geography education, directions in research and teaching, scientific discourse, HGD Symposium

\section{Einleitung}

Eine erste von Ingrid Hemmer und Peter BagolySimó organisierte Fishbowl-Diskussionsrunde zur Frage ,Geographiedidaktische Forschung - quo vadis?" auf dem Deutschen Kongress für Geographie in Berlin (2015) zeigte die verschiedenen Grenzlinien hinsichtlich theoretischer Ausrichtungen, Generationenkonflikten und hierarchischen Machtpositio-

Der Artikel wurde von beiden Autorinnen zu gleichen Teilen erarbeitet.

2 Die Namen der Beitragenden sind in der Reihenfolge ihres erstmaligen Auftretens im Beitrag genannt. nen innerhalb der Geographiedidaktik deutlich auf (vgl. Bagoly-Simó \& Hemmer 2016). Die Autorinnen dieses Beitrags beabsichtigten im Rahmen einer Fishbowl-Diskussion (II) auf dem HGD-Symposium in Jena (2017) die Diskussion in Bezug auf die aktuellen und zukünftigen Forschungstrends in der Geographiedidaktik in neuer Besetzung des inneren Diskutantenkreises fortzuführen.

Die Veranstalter/innen des HGD-Symposiums in Jena hatten die zweitägige Tagung unter das Motto ,Grenzen markieren - Grenzen überschreiten' gestellt (vgl. Dickel et al. 2018). Wir ließen uns vom Motto des Jenaer Symposiums leiten und luden Geographie- 
didaktiker/innen ein, bestehende Grenzen (die eigenen eingeschlossen) offen anzusprechen, diese so weit wie möglich wertfrei zu markieren, auch Wege jenseits von Grenzen aufzuzeigen, um in der schließlich fast zweistündigen Diskussion genau diese Konstruktionen wieder in Frage zu stellen und möglicherweise auch aufgezeigte Grenzen diskursiv zu überwinden. Zur Unterstützung der Diskussion sammelten wir von den eingeladenen Teilnehmer/innen je eine These im Vorfeld ein. In der Formulierung der Thesen hatten die Teilnehmer/innen freie Hand. Wir zielten auf eine möglichst diskussionsanregende Aussage ab, die eine spezifische Forschungsperspektive oder auch einen provokativen Ansatz in die Diskussion einbringen würde, sodass der inhaltliche Austausch ausgehend von den Thesen angeregt werden und sich verselbstständigen könnte. Die Fishbowl-Diskussion sah ähnlich wie in Berlin vor, Thesen zunächst im inneren Kreis zu diskutieren und in einem zweiten Schritt die Diskussion für Beiträge und Nachfragen aus dem Publikum zu öffnen.

Mit Hilfe eines, graphic recording' unterstütze Johanna Benz die Diskussion im Hintergrund und gab gleichzeitig den Anlass, aus der inhaltlichen Auseinandersetzung der Teilnehmenden eine ,Forschungslandkarte entstehen zu lassen. Die sukzessiv entstehende Darstellung der Diskussionsbeiträge und des Verlaufes diente nicht nur als Gedankenstütze, um im Anschluss an die Fishbowl-Runde die Teilnehmer/innen zu weiteren Diskussionen anzuregen. Auch während der Diskussion verfolgten die Anwesenden gespannt, was und vor allem wie Johanna Benz die inhaltliche Auseinandersetzung und verbalen Beiträge in einer graphischen Repräsentationsform dokumentierte. Die inhaltlichen Erläuterungen sollen daher auch in diesem Beitrag graphisch begleitet werden von den Insitu-Illustrationen bzw. dem großformatigen graphic recording der jeweiligen Thesen an der Plakatwand, die hinter der Diskussionsrunde aufgebaut war. Damit zieht sich auch ein visuelles Leitmotiv durch den gesamten Beitrag hindurch, das nicht nur der Veranschaulichung und Illustration dient, sondern auch als mögliche Quelle der Auseinandersetzung durch Irritation und Perspektivenwechsel. Weiter gefasst kann das graphic recording vielleicht sogar selbst als eine Methode verstanden werden, die einen Raum bietet, etwa für eine innovativ-kreative Fortführung des wissenschaftlichen Diskurses, der sich anderen, nicht textgebundenen Formen der Repräsentation von Geographie/-didaktik in Wissenschaft und Praxis öffnet. Anknüpfungspunkte an dieses Argument sehen wir in den Thesen von Uwe Schulze, Marion Plien und Eva Nöthen, die unter Kapitel 3 näher ausgeführt sind.

\section{Intention und Aufbau des Beitrags}

Der Hauptteil des Artikels basiert auf der inhaltlichen Aufarbeitung der aufgezeichneten und transkribierten Fishbowl-Diskussion in Jena. Hierbei versuchen wir einen schwierigen Spagat: Einerseits wollen wir die Dynamik des Diskussionsverlaufs mit Hilfe des Transkripts so nahe, wie das nachträglich möglich ist, am Original darstellen. Andererseits räumten wir den Diskussionsteilnehmer/innen die Möglichkeit ein, ihre eigenen, notwendigerweise von uns gekürzten Redebeiträge nachträglich für die Veröffentlichung der Fishbowl-Diskussion in Jena zu redigieren und den vollständigen Text vor der Einreichung noch einmal gegenzulesen. Die Möglichkeit der nachträglichen Textüberarbeitung nutzten die Diskutierenden in sehr unterschiedlicher Art und Weise. Während es einigen ein Anliegen war, möglichst nahe an der mündlichen Äußerung zu bleiben, um den ursprünglichen Charakter und die Dynamik der Diskussionsrunde nachzuempfinden, entschieden sich andere für eine intensivere sprachliche Überarbeitung ihrer Argumente. Daraus ergeben sich sehr unterschiedliche Sprachstile in den Beiträgen, die keinen Rückschluss auf die tatsächliche rhetorische Gestaltung der Diskussion mehr erlauben. Zugunsten einer höheren inhaltlichen Klarheit erscheint uns diese Vorgehensweise jedoch legitim. Darüber hinaus konnten die Diskutierenden auch überprüfen, in welchem Kontext die eigenen Passagen eingebettet wurden, was eine interessante Diskussion mit und unter den Autorinnen des Beitrags anregte. Diese Methode der Textproduktion und -redaktion lehnt sich an Ideen des "kollaborativen Schreibens" (Lowry et al. 2004) an, aus der wir einige Elemente in den Schreibprozess dieses Artikels integrierten, wie z. B. das Einbeziehen mehrerer Personen in die Erstellung und Überarbeitung eines Textes, das Verhandeln von verschiedenen Positionen, Perspektiven und Meinungen und deren angemessene Repräsentation im Text, das Koordinieren von verschiedenen Arbeitsfortschritten und Rückschlägen sowie eine dynamische Fluktuation unter den Teilnehmer/innen (vgl. im engl. Original ebd.: 71). Weil wir bei diesem unserem Versuch des ,kollaborativen Schreibens' als erkennbare Autorinnen noch sehr deutlich die Fäden (v. a. bei der Textkoordination) in der Hand hielten, möchten wir lieber von einem ,kooperativen Schreibprozess' sprechen.

Weitaus mutigere Beispiele finden sich in der anglo-amerikanischen Sozialgeographie. Drei Beispiele: Caitlin Cahill (2004) lotete die Grenzen der partizipatorischen Forschung mit Jugendlichen als, co-researchers ' und, co-writers of research' in New York aus. Janet G. Townsend et al. (1995) verliehen den Narrationen und Anliegen von ,Pionier-Frauen' auf der ganzen Welt anhand von ausgewählten lokalen Beispielen 
(u. a. Frauen, die an Siedlungsprojekten im kolumbianischen Amazonas-Regenwald beteiligt waren) eine Stimme in der internationalen Fachliteratur. In einer erst kürzlich publizierten Forschungsarbeit berichten Kath Browne et al. (2017) von einem interdisziplinären und transnational verorteten (d.h. ,multi-sited) Forschungsteam, das sich der Herausforderung stellte, einen kollaborativ geschriebenen Beitrag zu,transnational feminist queer methodologies 'zu erarbeiten, der auf den eigenen Dialogen zum Forschungsprozess basierte und multi-medial sowie sozio- technisch unterstützt wurde (z.B. durch Skype-unterstützte Gruppengespräche, Kommunikation auf $A p p$-basierenden Plattformen sowie Face-to-face-Austausch). Beispiele in der fachdidaktischen Literatur zu ,kollaborativem Schreiben' finden sich u. a. in der Geschichtsdidaktik (vgl. Hodel \& Haber 2007; Lohse \& von Buchholz 2007).

Von der Vorgehensweise eines, kooperativen Schreibens' inspiriert, beabsichtigten wir einen respektvollen gedanklichen Austausch unter Geographiedidaktikerinnen und -didaktikern zu etablieren und mit dieser Methode auch inhaltlich weiter auszuloten, wie wir in diesem Prozess bestehende Grenzen im Denken und Schreiben von und über Geographiedidaktik überschreiten könnten.

Den Teilnehmenden am HGD-Symposium in Jena werden bei der Lektüre dieses Beitrags gewisse Leerstellen und Lücken auffallen. Um Missverständnissen vorzugreifen, möchten wir hierauf eingehen. Die Idee, die Fishbowl-Diskussion nachträglich zu veröffentlichen, wurde von der Mehrheit der Diskutierenden begrüßt und konstruktiv unterstützt. Dieser Prozess der gemeinsamen Textproduktion und -redaktion hat nicht nur sehr viel Freude gemacht, sondern war auch mit einem beträchtlichen Arbeitsaufwand verbunden, der von einem intensiven inhaltlichen Austausch begleitet und bereichert wurde. Es ist uns wichtig herauszustellen, dass etwaige ,Lücken' und möglicherweise überrepräsentierte Diskurse in der schriftlichen Fassung der Diskussion nicht auf unsere Zensur oder Korrektur bestimmter Aussagen und Stimmen zurückzuführen sind. Wir haben uns bemüht, alle wesentlichen Diskussionsstränge zu erfassen. Allerdings gab es neben den im Text repräsentierten Stimmen und Argumenten noch weiterführende Gedanken, auf die wir auf Wunsch einiger Diskutierender nicht näher eingehen können. Die jeweiligen Personen distanzierten sich von einer Veröffentlichung ihres mündlichen Diskussionsbeitrags, obgleich allen Beteiligten, wie eingangs beschrieben, die Option offenstand, ihre Äußerungen nachträglich für eine Publikation zu überarbeiten. Dies finden wir sehr bedauerlich, akzeptieren und respektieren jedoch die Entscheidung derjenigen, die ihre Stimmen und Argumente aus der Veröffentlichung zurückgezogen haben.
Von den Höhen und Tiefen während des aufwändigen Entstehungsprozesses dieses Artikels weniger entmutigt als motiviert, möchten wir mit diesem Beitrag gerne eine Diskussion zur Frage anregen, ob es vor dem Hintergrund der von uns gewählten Methoden der Diskussion und anschließenden Textproduktion so etwas wie eine ,formsensible', sprachsensible ${ }^{\text {'3 }}$ und ,gendersensible' Geographiedidaktik geben könnte. Konkreter fragen wir uns, wie eine Geographie bzw. Geographiedidaktik aussieht, die sich nicht nur um die sprachliche Formulierung ihrer textlich transportierten Inhalte kümmert, sondern sich auch Gedanken über neue Formen der visuellen, auditiven, karto-/ graphischen, haptischen Formen der Repräsentation von Geographie macht. Mit dem Schlagwort ,sprachsensible' Geographiedidaktik greifen wir bereits etwas auf die textliche Ebene der Fishbowl-Diskussion vor, deren Dokumentation anders als klassische schriftsprachliche Publikationen gelagert ist. Mit Formsensibilität adressieren wir deren methodische Visualisierung. Die Gendersensibilität sollte sowohl durch die Auswahl der Eingeladenen gestärkt und in der Verschriftlichung der Diskussionsbeiträge auch sprachlich berücksichtigt werden.

Im vorliegenden Artikel unternehmen wir vor dem Hintergrund und der Methodik seiner Entstehung eine vorsichtige Annäherung an die hohen Ansprüche der Form-, Sprach- und Gendersensibilität, was implizit auch den Aspekt der Machtsensibilität einschließt. Einen ersten Entwurf für ,machtsensible geographiedidaktische Konzepte' legte Birte Schröder (2016) am Beispiel des interkulturellen Lernens dar und lotete Potenziale einer postkolonialen Perspektive für den Geographieunterricht aus. In der Vorbereitung dieser Publikation haben wir sowohl die Vorzüge als auch die Fallstricke einer, multisensiblen Geographiedidaktik' und eines kooperativen Schreibprozesses kennengelernt.

Wir sind uns beispielsweise durchaus bewusst, dass diese einleitenden Gedanken zur Fishbowl-Diskussion hier eine Metaebene einnehmen, die wir so in unseren möglichst neutral gehaltenen Moderationsrollen in Jena (noch) nicht geäußert haben. Geographiedidaktik weiter zu denken, ist für uns ein kontinuierlicher Prozess und eine intellektuelle Herausforderung, die nicht künstlich zu einem bestimmten Zeitpunkt oder in einem eingegrenzten Denkraum eingefroren werden sollte.

\footnotetext{
$\mathrm{Zu}$ sprachsensiblem Geographieunterricht gibt es bereits geographiedidaktische Forschungsarbeiten, u. a. von Alexandra Budke, Miriam Kuckuck und Günther Weiss (siehe Budke \& Weiss 2014; Budke \& Kuckuck 2017). In unserem Beitrag geht es uns jedoch vor allem um den Aspekt der ,Sprachsensibilität', in welcher sprachlichen Form und Verpackung also bestimmte Inhalte in Bezug auf Geographie, Geographiedidaktik und Geographieunterricht repräsentiert werden.
} 
Der chronologischen Dramaturgie entsprechend möchten wir die Dokumentation der Diskussion gerne mit dem Impulsreferat von Ute Wardenga ${ }^{4}$ einleiten.

\section{3 „Hier ist etwas in Bewegung!“ - Auftaktreferat zu den Thesen der Geographiedidaktiker/innen}

Aus ihrer eigenen Position als Wissenschaftshistorikerin fügte Ute Wardenga die eingesandten Thesen der Geographiedidaktiker/innen zu einer Narration bzw. ,Geschichte' zusammen (siehe Dickel et al. 2018). Analog zum Motto des HGD-Symposiums markierte sie nicht nur die Grenzen der aktuellen Geographiedidaktik, sondern bot auch Denkanstöße für die Fishbowl-Diskussion an, um diese Grenzen im Dialog miteinander zu überwinden. Wir nehmen das Impulsreferat von Ute Wardenga in leicht gekürzter Form hier auf und verknüpfen die schriftlichen Thesen mit ihrer Eingangsrede. Hierbei bediente sich Ute Wardenga - mit beiläufiger Selbstironie - einem altbekannten Prinzip der Geographiedidaktik: vom Nahen zum Fernen. Sie bezog zunächst Stellung zu Thesen, die ihr aus der Perspektive ihres eigenen Forschungsschwerpunkts naheliegender erschienen, um sich im Weiteren mit Thesen auseinanderzusetzen, die ihr spannende Anregungspunkte für eine gedankliche Annäherung gaben oder sie dazu einluden ,produktive Missverständnisse' zu riskieren. Im Folgenden sind Ute Wardengas Überlegungen, die sie im Zusammenhang mit den eingereichten Thesen äußerte, wiedergegeben:

Die geographiedidaktische Forschung bleibt trotz einer wachsenden Zahl englischsprachiger Publikationen in ihrer historisch ererbten nationalen Tradition verhaftet. Vor dem Hintergrund eines wieder erstarkenden Nationalismus halte ich eine grundlegende international angelegte Forschungsdiskussion über die Rolle des Geographieunterrichts im Zeitalter globalisierter und transnationaler Lebenswelten für wünschenswert. (Holger Jahnke)

Holger Jahnke schlägt vor, in den geographiedidaktischen Forschungen den Nationalstaatscontainer $z u$ verlassen, um zusammen mit Kolleginnen und Kollegen aus dem internationalen Bereich die Rolle von Geographieunterricht im Kontext der Globalisierung, und das ist wichtig, und die durch diesen Kontext der Globalisierung produzierten transnationalen Lebenswelten neu zu denken. Ich verstehe die These in zweierlei Hinsicht:

Das Eingangsreferat von Ute Wardenga sowie das AbschlussStatement von Ralf Koerrenz sind im Tagungsband des HGD-Symposiums in ungekürzter Form publiziert (siehe Dickel et al. 2018).
Erstens als einen starken Hinweis darauf, dass Didaktik Antworten geben sollte auf die Herausforderungen des Geographieunterrichts vor Ort. Zum Beispiel: Wie gehen wir produktiv mit der sozio-kulturellen Vielfalt unserer Schüler/innen um? Zweitens verstehe ich die These als Aufforderung, die gesuchten Antworten durch gezielten Aufbau internationaler Forschungsnetzwerke zu finden, um dann in diesen internationalen Forschungsnetzwerken gemeinsam gegen den aktuell, global wieder erstarkenden Nationalismus anzugehen. (...) Und die Erfahrung sagt, Gemeinsamkeit lässt sich auf solchen internationalen Plattformen erst dann herstellen, wenn nämlich die Partner/innen im Spiegel der je anderen Selbstreflexion üben und sich der Einsicht öffnen, dass ihre eigene Weltwahrnehmung nicht die einzig mögliche, die einzig richtige und die einzig natürliche ist.

Angesichts zunehmender globaler Disparitäten und einer interessensgeleiteten bis legitimatorischen Behandlung im öffentlichen Diskurs bedarf es einer geographiedidaktischen Forschung, die gängige Erklärungsansätze und Weltsichten hinterfragt und ihre Repräsentationsformen in der geographischen Bildung analytisch reflektiert - etwa entlang der Verwendung von Begriffen wie Demographie, Globalisierung, Kulturdifferenz und Nachhaltigkeit. (Leif Mönter)

Die notwendige Vertiefung zu Holgers These hat wunderbar komplementär Leif Mönter formuliert. Er fordert geographiedidaktische Forschung auf, ihre vorhandenen Erklärungsansätze und Weltsichten sowie deren Repräsentationsformen stärker als bisher analytisch zu reflektieren. Die These knüpft an Basispraktiken des Wissenschaftler/inseins an und sie ist insbesondere für jüngere Wissenschaftler/innen meiner Meinung nach attraktiv, weil sie nämlich implizit auch als Aufforderung zur kritischen Distanzierung vom Hergebrachten verstanden werden kann und so Potenziale für die Entfaltung eines wissenschaftlichen, eines eigenen Selbst gegen eine Welt der Altvorderen enthält. Das ist wissenschaftssozial absolut notwendig, denn der Nachwuchs braucht Raum, um Ideen entfalten zu können, weil sich ein soziales System letztlich nur durch seine Fähigkeit zum Wandel auf Dauer stellen kann. Dieser Wandel wird allerdings meiner Meinung nach nicht ganz einfach sein.

Um der gestiegenen gesellschaftlichen Relevanz von Geotechnologie in Bildungsprozessen wirkungsvoll begegnen zu können, benötigen wir einen Paradigmenwechsel des GIS-Bezugs im Unterrichtsgeschehen, respektive in der geographischen Lehramtsausbildung: Weg vom tradierten Verständnis von GIS als „Forschungswerkzeug der Geographie" der 1990er Jahre hin zum lebensweltlichen Bezug digitaler Geomedien als neue Formate der Web-basierten Kommunikation räumlicher Informationen. (Uwe Schulze)

Denn komplementär zu Leifs These steht die These von Uwe Schulze. Sie lässt mit Blick auf GIS eine 
offensichtlich vorhandene Diskrepanz von System und Lebenswelt anklingen und geht, jetzt radikalisiere ich, an die bislang, taken for granted" genommenen Wahrheitsansprüche von symbolisch kodierten Medien der Visualisierung, vulgo Karten. Nach meinen eigenen Forschungen haben wir es hier mit dem Kernelement einer bereits im späten 19. Jahrhundert global durchgesetzten Praxis des Kartenherstellens und des Kartengebrauchs zu tun, die für den Geographieunterricht absolut konstitutiv ist. Denn Geographie ist weltweit das einzige Schulfach, in dem Schüler/innen diese für die Moderne wichtigen Kulturtechniken lernen. Das scheint für viele Geographinnen und Geographen bis heute so selbstverständlich, dass sie ihre mit dem Ziel der Herstellung von Evidenz produzierten Karten bis in die jüngste Vergangenheit hinein für die wahre Abbildung, der' Wirklichkeit hielten. Von diesem Wahrheitsbegriff rückt Uwe Schulze, so weit ich das sehe, nicht im Grundsatz ab, das ist auch gut so. Denn auch in Zukunft wird Geographieunterricht wahrscheinlich nicht ohne, facts und figures' auskommen. Gleichwohl, und das ist für mich der Kern der These, ist eine bessere Anknüpfung an die Lebenswelten und Praktiken der, digital natives' unumgänglich. Wenn man das bei allen derzeit noch im Wege stehenden finanziellen Implikationen, insbesondere in der Ausstattung der Schulen, umsetzen kann, dann werden die Anforderungen bezüglich eines Unterrichts in Medienkompetenz ganz enorm wachsen.

Mediale Repräsentationen und Medienerfahrungsräume sollten stärker in den geographiedidaktischen Forschungen berücksichtigt werden. (Marion Plien)

Und genau hier setzt die These von Marion Plien 5 an. Sie fordert eine stärkere Berücksichtigung von medialen Repräsentationen und Medienerfahrungsräumen in der geographiedidaktischen Forschung. Dabei legt sie reflexiv den Finger auf die Wunde, indem sie (...) verdeutlicht, dass Medien eben nicht mehr als reines, das heißt verbindliche Wahrheiten transportierendes Fenster der Welt oder aber als Abbild von Untersuchungsgegenständen gesehen werden können, sondern Eigenwirklichkeiten erschaffen und subjektive Erfahrungsräume anbieten, die es im Unterricht zu reflektieren gilt.

Bei der Vorbereitung wusste ich nicht, wie jung die Didaktik mittlerweile ist! (...) Spätestens jetzt werden einige von Ihnen in stiller Verzweiflung gedacht haben, "Himmel, was sollen wir eigentlich alles noch machen?" Denn wenn man es genau reflektiert, dann provozieren alle bisher interpretierten Thesen auf ihre je eigene Art vorhandene Grenzen. (...)

\footnotetext{
Marion Plien konnte an der Fishbowl-Diskussion in Jena krankheitsbedingt nicht teilnehmen. Ihre eingereichte These (und eine umfassende schriftliche Erläuterung dazu) wurden trotzdem in die Diskussion einbezogen.
}

Mehr Mut zur Kunst: Im Sinne des, artistic research', der Wissenschaft und Kunst gleichermaßen als Prozesse der Erkenntnisgewinnung und -vermittlung versteht und zu integrieren sucht, ruhen in der Einbindung künstlerischer Praktiken in die geographiedidaktische Forschung noch ungehobene Schätze. (Eva Nöthen)

Eva Nöthen fordert "Mehr Mut zur Kunst!“ und ermuntert so zwei in der funktionalen Differenzierung moderner Gesellschaft, und ich bleibe jetzt im Bereich der Systemtheorie, getrennte soziale Systeme, Wissenschaft einerseits und Kunst andererseits, in der geographiedidaktischen Forschung, (...) wieder näher aneinander zu rücken und miteinander in Beziehung zu setzen. Das provoziert ganz erheblich die selbst geschaffenen Grenzen des Wissenschaftssystems, das seine Eigenständigkeit gerade dadurch erlangt hat, dass es eben nicht mehr mit Kunst verwechselt oder in Zusammenhang gebracht werden durfte. Die These von Eva kann insbesondere für Kolleginnen und Kollegen aus der physischen Geographie oder der quantitativen Lehr-Lern-Forschung eine Herausforderung darstellen.

Die Konditionierung der Geographiedidaktik auf Fragen unterrichtspraktischer Verwertbarkeit verhindert kreative Forschung. Wir brauchen eine Emanzipierung unserer Forschung vom Geographieunterricht, um geographische Bildung anders denken und gestalten zu können. (Verena Schreiber)

Sich herausgefordert zu fühlen scheint mir aber auch noch viel mehr bei der These von Verena Schreiber der Fall zu sein. Mit provozierender Metaphorik ist sie im besten Pawlowschen Sinne der Meinung, dass die Konditionierung der Geographiedidaktik auf Fragen unterrichtspraktischer Verwertbarkeit kreative Forschung behindere und deshalb eine Emanzipierung von Geographieunterricht nötig sei. Damit operiert diese These ganz hart an der Identitätsgrenze älterer Didaktikerl innen, denn diese können sich nur zu gut daran erinnern, welch harte Kämpfe nötig waren, um Geographiedidaktik als eigenständiges Feld der Expertise zu etablieren, in das die Fachwissenschaft gefälligst nicht hineinzureden hatte (...).

Vom Geographie Unterrichten zum Lernen mit Geographie. (Thomas Jekel)

Ehe wir in die Diskussion der Thesen eintreten, deshalb ein paar abschließende Bemerkungen, die ich mit Hilfe der These von Thomas Jekel formulieren möchte. Also für mich als Außenstehende ist diese These ziemlich apokryph dahergekommen. Ich habe mir rein gar nichts darunter vorstellen können, und mir gedacht, dass sie ja gerne Anlass für produktive Missverständnisse geben könnte (...), die wir gerne gemeinsam klären können. 
Ihr Impulsreferat schloss Ute Wardenga mit einem appellativen Fazit:

Mut zu Lernen und Entwicklungsfähigkeit wird meiner Meinung nach nötig sein für eine Geographiedidaktik, von der ich im Spiegel der hier skizzierten Thesen vermute, dass sie sich in einer Phase des Aufbruchs, der Reflexion ihrer Grundlagen, des Zweifels an der Tauglichkeit vorhandener Selbstbeschreibungen und tradierter Selbstverständnisse befindet. Das finde ich als Wissenschaftshistorikerin, die an der Geographiedidaktik auch fremdinteressiert ist, richtig toll und freue mich deshalb schon sehr auf die anschließende Diskussion.

\section{Die Autor/innen beziehen Stellung zu ihren Thesen}

Im stark eingeschränkten Rahmen eines Artikels können wir die inhaltliche und dynamische Entwicklung der Diskussion nicht in ihrem ganzen Umfang wiedergeben und sind gezwungen, eine gewisse inhaltliche Auswahl und argumentative Schwerpunktsetzung zu treffen. Trotzdem möchten wir uns an den Ablauf der FishbowlDiskussion anlehnen, der zunächst eine Diskussion der Thesen in der inneren Runde der eingeladenen Personen vorsah, bevor sich der Kreis für Stimmen aus dem Publikum öffnete. Auch wenn die Stellungnahmen in ihrem Umfang deutlich gekürzt werden mussten, ist die Aussagekraft des jeweils vorgebrachten Arguments maßgeblich und wird so weit wie möglich erhalten.

Leif Mönter: Ja, daran [an die Einleitung von Ute Wardenga] würde ich gerne anknüpfen und meine These erläutern, die freilich sehr kurz sein musste (...). Meine These sagte aus (...), dass in der stark von Disparitäten geprägten Gegenwart zahlreiche geographisch besetzte oder verwendete Begriffe auftauchen, aber häufig ohne als solche expliziert zu werden, sondern mit einem vorrangig legitimatorisch-politischen Charakter. Das können beispielsweise Begriffe, Narrationen oder vermeintliche Selbstverständlichkeiten sein wie Volk, Nation, Kultur, Globalisierung oder Demographie (...). Und deshalb glaube ich, dass es derzeit ein zentraler Auftrag des Faches ist, ideologiekritisch derlei Begriffe und die mit ihnen verbundenen Vorstellungen zu erörtern und dabei auch die Ausrichtung des Faches in Bezug auf seine Geschichte zu reflektieren. In den Vorträgen heute von H.-D. Schultz beziehungsweise Ute Wardenga wurde treffend ausgeführt, wie die Geographie sich gerade, wenn sie sich als un-oder überpolitisch verstanden hat, hat instrumentalisieren lassen bzw. sich selber angeboten hat, sich für politische Programmsetzungen oder Ideologien zu funktionalisieren. Natürlich ist das im Nachbinein, mit einem gewissen historischen Abstand, immer leichter zu identifizieren. Doch gerade in der heutigen Zeit wäre

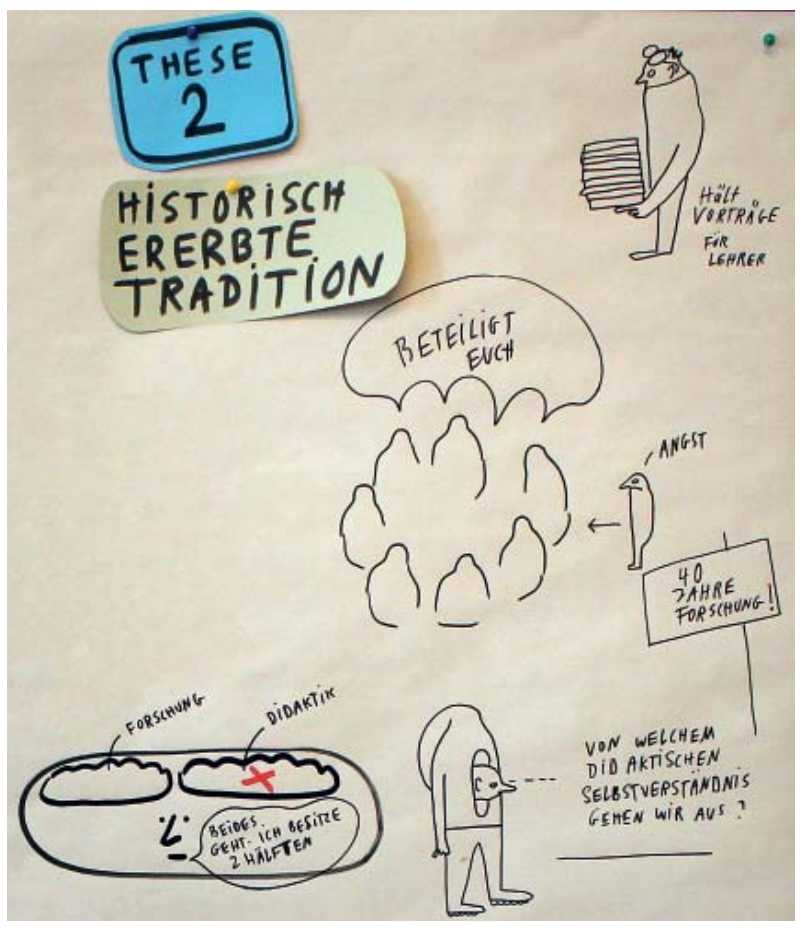

Abb. 1: Illustration der These von Leif Mönter und der zugehörigen Diskussion; Zeichnung: Johanna Benz.

es sehr wichtig, einmal grundsätzlich zu überprüfen, für welche Inhalte und Methoden, für welche empirischen Forschungsarbeiten Geographie und Geographiedidaktik eigentlich stehen. Das wäre eine spannende und selbstkritische Reflexion und könnte uns dazu anregen, einen analytischen Standpunkt auch in die eigene Forschung zu integrieren. Das heißt, dass wir nicht Begriffe als unumstrittene Zielsetzung verstehen, und uns fragen, wie wir diese beispielsweise messen oder die Kompetenzen dazu entwickeln können, sondern dass wir Begriffe einmal wirklich wieder stärker analysieren.

Die Frage nach der Bedeutung von Sprache (und ihren Bedeutungszuweisungen) für die Geographiedidaktik spielte auch im weiteren Verlauf der Diskussion eine wichtige Rolle. So wurde von einem Diskussionsteilnehmer der Gedanke aufgebracht, dass Sprachen Weltbilder und Wissenschaftskulturen repräsentieren und auch überrepräsentieren und daher ein Bewusstsein für die sprachlichen Grenzen und Möglichkeiten etwa in der internationalen Wissensgenerierung relevant ist. Uwe Schulze weitet den Gedanken auf Kartensprache als geographisches Arbeitsmittel und auch Identifikationsinstrument aus.

Uwe Schulze: An dieser Stelle möchte ich einfach mal anschließen, (...) weil hier ein Punkt angesprochen wird, der auch in meiner These einen sehr wichtigen Stellenwert hat. Das ist die Sprache, in der wir über die Welt reden. Hier spielen für mich (...) nicht nur klassische Karten, sondern (...) vor allem digitale kartographische Reprä- 


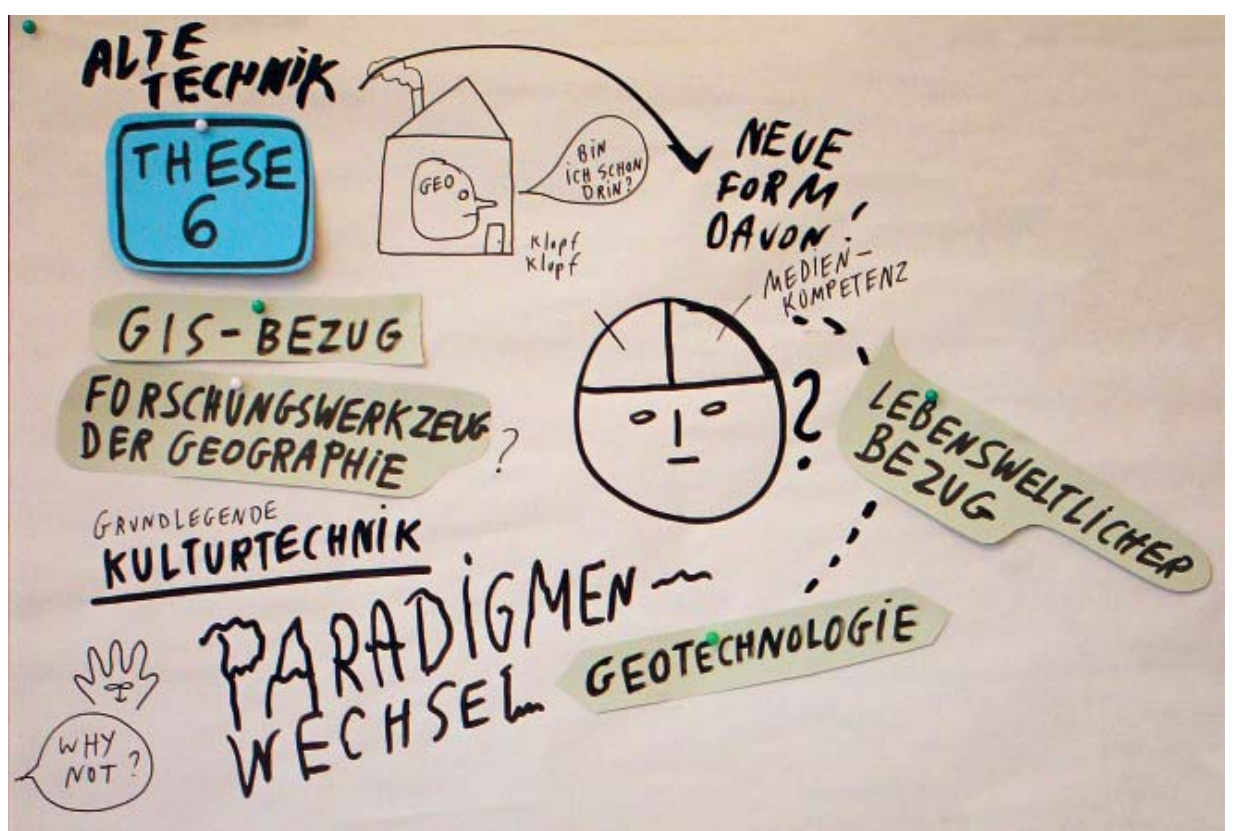

Abb. 2: Illustration der These von Uwe Schulze und der zugehörigen Diskussion. Zeichnung: Johanna Benz. sentationen und all das, was wir als digitale Artefakte in unserer alltäglichen Begegnung von Welt und Kommunikation finden, eine große Rolle. (...) Mit meiner These schließe ich auch an das, was Franke Kraas auf dem Deutschen Kongress für Geographie in Berlin adressiert hat hinsichtlich der Richtung, in die sich das Fach Geographie auch entwickeln muss: Im Kern: Wie sieht es eigentlich mit der Digitalisierung aus? Wie geht man mit dem Digitalen in der Geographie um? Wir schlagen uns in der Geographie noch immer, seit den 90er Jahren, mit diesem Schlagwort von GIS als, tradiertes Überbleibsel der Desktop-GIS-Entwicklung herum (...). Und wenn wir uns die internationale Forschung anschauen, sehen wir, dass gerade im angloamerikanischen Bereich die Diskussion mindestens 10, 15 Jahre weiter ist und wir dort eben nicht mehr nur von GIS als einer methodischen Idee sprechen, (...) sondern von, geospatial technologies', im Sinne einer eigenständigen Domäne. Und an dieser Stelle ist für mich daher die Frage zentral, wo sich da die Geographie verortet, denn die Geographie hat keinen Allgemeinanspruch auf, Raum' oder, geospatial'. Die, geospatial domain' ist ein multidisziplinäres Gebiet, das, ganz in diesem Sinne, von keinen fachlichen Grenzen eingeschränkt wird. Wenn wir uns diese Domäne als zentralen (...) Gegenstandsbereich vorstellen wollen, (...) müssen wir überlegen, wie wir mit dieser neuen Art der Sprache umgehen wollen, die in der Lebenswelt von Kindern und Jugendlichen, von Schülerinnen und Schülern, aber eben auch von Lehrerinnen und Lehrern präsent ist. Auch müssen wir uns klar werden, wie wir mit der Digitalisierung, die an die Tür klopft bzw. die längst da ist, auch in Zukunft umgehen wollen. Wir müssen die damit einhergehenden, vielen verschiedenen Aspekte, angefangen von der Medienkompetenz (...), über die inhaltlichen Perspektiven hin bis zum Lernen in der Geographie umsetzen.
Den in diesem Sinne also mehrfach eingebrachten Gedanken, welchen Einflüssen Erkenntnisproduktion unterliegt, greift Eva Nöthen auf und bringt Kunst als mögliche Blickerweiterung oder auch Grenzüberschreitung in den Erkenntnisprozess ein.

Eva Nöthen: Mit meiner These knüpfe ich an einer etwas anderen Stelle an (...). Mir geht es darum, den Fokus stärker auf uns selbst als Forschende zu richten, $z u$ überlegen, mit welcher Haltung wir eigentlich an das Objekt, das wir beforschen, herantreten. Vor diesem Hintergrund ist es mir wichtig, den Blick zu weiten und dafür zu sensibilisieren, wo in unseren Forschungsprozessen die Grenzen zwischen Wissenschaft und Kunst und damit - im postmodernen Sinne - zwischen normativen und ästhetischen Betrachtungsweisen durchlässig werden. Diese Herangehensweise führt neben inhaltlichen nicht zuletzt zu selbstreflexiven Fragen wie: Welche ist meine individuelle Haltung zum Forschungsgegenstand? Was ist mein persönliches Interesse und auch meine Motivation, mich mit ihm auseinanderzusetzen? Ich glaube, dass der , artistic research' - eine wissenschaftstheoretische Bewegung, die in den letzten 10 bis 15 Jahren erstarkt ist und eine Synthese von künstlerischen und wissenschaftlichen Ansätzen verfolgt - großes Potential birgt. Die Synthese von künstlerischen und wissenschaftlichen Ansätzen soll nämlich dazu führen, ästhetische Erfahrungen stärker in wissenschaftliche Forschungsprozesse einzubeziehen oder umgekehrt ein ästhetisches Forschen stärker zu systematisieren, indem wissenschaftliche Ordnungssysteme aufgegriffen werden. Die zentralen theoretischen Impulse kommen hierzu aus der Kunstwissenschaft. Damit erweist sich die Kunst derzeit deutlich offener, die ordnenden Prinzipien der Wissenschaft aufzugreifen und in einem kreativen Gestaltungsprozess die persönliche Be- 


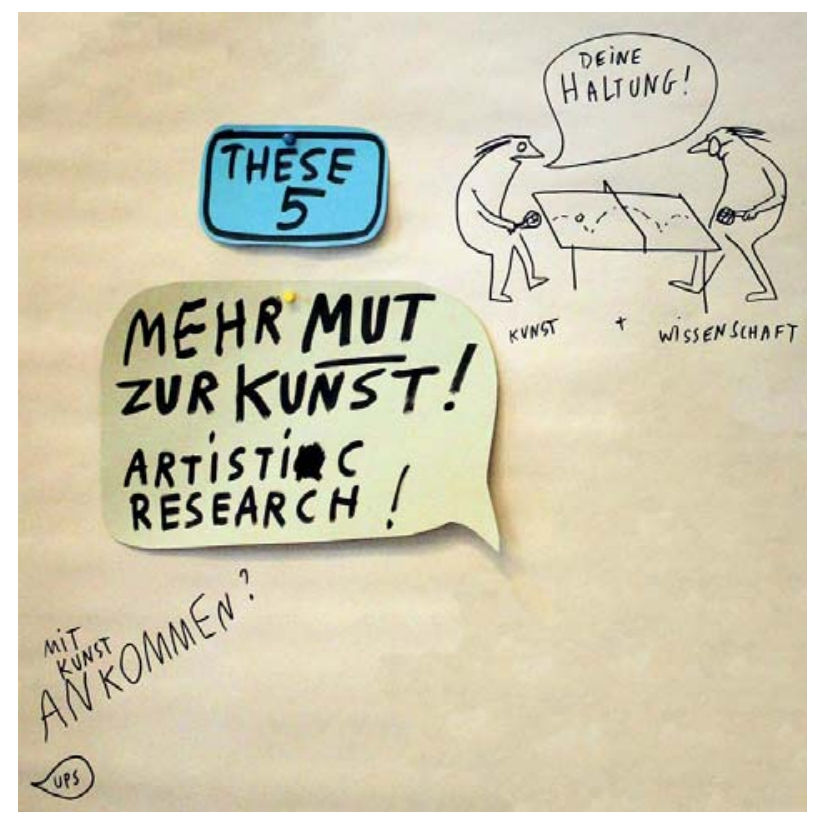

Abb. 3: Illustration der These von Eva Nöthen und der zugehörigen Diskussion; Zeichnung: Johanna Benz.

gegnung mit dem Forschungsgegenstand und das Erleben des Forschungsprozesses aktiv mit einzubinden. Ich bin jedoch überzeugt, dass es auch für Wissenschaftler/innen in unserem Fach sehr bereichernd sein kann, Forschungsprozesse kreativer und freier zu denken: Das, womit wir uns beschäftigen, persönlich wirken zu lassen, um aus der persönlichen Begegnung, dem persönlichen Erleben heraus zur disziplinären und gesellschaftlichen Relevanz des Themas zu finden.

Verena Schreiber: Ich kann mich sehr gut an viele Dinge anschließen. Ich habe die Aufforderung zur Diskussion jedoch auch so verstanden, dass die Thesen durchaus provokativ sein dürfen, um eine Debatte zu entzünden. Das erklärt vielleicht meine wahrscheinlich wirklich extrem provozierend wirkende These, die auf den zweiten Blick vielleicht gar nicht so provozierend ist, (...) weil es nicht meine Intention ist, in Richtung einer Trennungs- oder Grenzziehungsdebatte einzutreten zwischen Fachdidaktik und Fachwissenschaft. Das sind Grenzerfahrungen, die ich persönlich aus der eigenen Wissenschaftssozialisierung mit mir selber ausmachen muss. (...) Mit dem Übergang von meiner fachwissenschaftlichen Sozialisierung vor etwa zwei Jahren in die Fachdidaktik stelle ich fest, dass dieser Übergang eine enorme Herausforderung ist und bei mir wahnsinnig viele neue Denkprozesse ausgelöst hat. Das ist eine durchaus positive Bewertung der Fachdidaktik, die mich zu ganz neuen Fragen bringt. (...) Ich habe vorher in der Schule gearbeitet, allerdings aus einer fachwissenschaftlichen Sicht. Und auf einmal werden mir durch diesen neuen fachdidaktischen Bezug auf Schule die Bedingungen und Möglichkeiten vorgegeben, aus denen meine eigene Existenz nun, durch diesen Bezug auf Schule, möglich sein darf. Daher frage ich immer: "Kann das nicht auch andersrum sein?". In diese Richtung sollte die These gehen (...): Sollte der Bezug auf Schule uns die Bedingungen tatsächlich vorgeben, unter welchen die eigene Existenz als Fachdidaktikerinnen und Fachdidaktiker möglich sein darfoder sollte das umgekehrt sein? Das ist vielleicht eine Frage, mit der sich auch viele Fachdidaktiker/innen hier rumschlagen - dieser Bezug zwischen Schule und Fachdidaktik, nicht zwingend zwischen Fachwissenschaft und Fachdidaktik. Gibt der Bezugsrahmen zur Schule mir nun die Möglichkeiten und Perspektiven vor, unter denen ich forschen darf als Fachdidaktikerin? Gibt mir die Geographiedidaktik die Denkräume und Möglichkeiten vor, in denen ich forschen darf? Und es stellt sich für mich die Frage: Muss das so sein? (...) Kann das nicht eigentlich anders herum sein, (...) dass die didaktische und fachwissenschaftliche Forschung die Bedingungen vorgibt, unter denen eine schulische Existenz oder

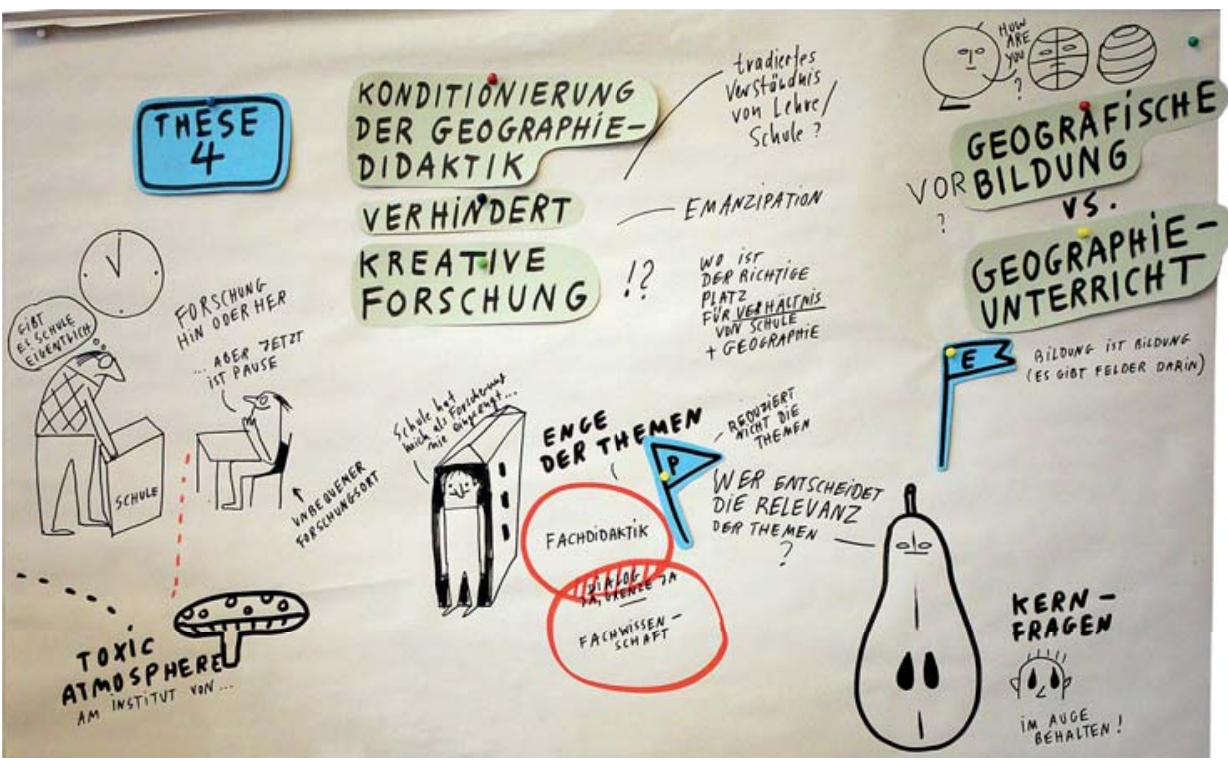

Abb. 4: Illustration der These von Verena Schreiber und der zugehörigen Diskussion; Zeichnung: Johanna Benz. 
schulische Unterricht möglich sein darf? Das ist die Umkehrung. (...) Was mir wichtig wäre: Denken wir doch einmal grundsätzlich über das Verhältnis von Schule und geographiedidaktischer Forschung nach. (...) In welchem Verhältnis stehen diese eigentlich?

Uwe Schulze setzt an dieser Stelle an und öffnet angesichts der Frage nach dem Einfluss auf Schule die Perspektive erneut für methodische Aspekte des Geographieunterrichts. Er stellt die Frage, inwieweit und vor allem wie auch alltagsweltliche Anregungen für Bildungsprozesse in Wert gesetzt werden können. Er erläutert dieses Argument am Beispiel von aktuellen Geotechnologien. Damit könnte die Frage nach den Bildungszielen und dem Bildungsauftrag von Schule erweitert und gleichzeitig der Anspruch an die Schule eingelöst werden, adäquat und zukunftsgerichtet auf das Leben und Lernen außerhalb der Schule vorzubereiten.

Uwe Schulze: Das aktuelle, Methodik-orientierte Unterrichten von GIS geht vor dem Hintergrund der veränderten soziotechnischen Entwicklung der GI-Technologie mittlerweile völlig an der Lebenswelt vorbei, so dass man fragen muss, nicht was der methodische Sinn und Zweck dieses spezifischen Instruments im Unterricht ist, sondern was eigentlich der Bildungsgehalt ist, was das Spezielle daran ist, dass wir uns mit diesen Medien, digitalen Medien überhaupt (...) auseinandersetzen.

\section{Der innere Kreis der Fishbowl-Runde öffnet sich für Meldungen aus dem Publikum}

\subsection{Unbequeme Grenzgänge zwischen Fachdi- daktik, Fachwissenschaft und der Praxis von Forschung und Unterricht}

Im Folgenden lassen sich mehrere Debatten nachzeichnen. Wir haben uns hier erlaubt, die Chronologie in Teilen aufzubrechen, um Argumente, die sich aufeinander beziehen, der Lesbarkeit halber im Verbund darzustellen.

Die Frage nach dem Verhältnis von Schule, Unterricht und geographiedidaktischer Forschung provozierte spontane Wortmeldungen aus dem Publikum.

Einerseits wurde die These des Auseinanderdriftens von Fachwissenschaften und Fachdidaktik aufgestellt. Der extrem ausgeprägte Spezialisierungstrend in den Fachwissenschaften, der durch einen Geographiedidaktiker moniert wurde, kann sicher zu Recht kritisch bewertet werden. Die Vertiefung der Argumentation mündete jedoch in eine grundsätzliche Infragestellung insbesondere einiger humangeographischer Forschungsrichtungen (wie z. B. ,blutjunger' feministischer Geographien) mit ihren je spezifischen Themen und Methodologien. Von dieser Position möchten wir uns abgrenzen, weil sie den notwendigen theoretischen und methodologischen Perspektivwechsel, der mit einem jeweils anderen Forschungsansatz verbunden ist, verweigert. Grenzen zu markieren ist eine Sache, Grenzüberschreitungen benötigen jedoch eine grundsätzliche Offenheit und akademische Toleranz Andersdenkenden und Andersforschenden gegenüber. Diese Überlegung impliziert für die Geographiedidaktik auch, sich unvoreingenommen mit aktuellen Forschungstrends und Themen in der Fachwissenschaft auf nationaler und internationaler Ebene auseinander zu setzen, um mögliche Anschlüsse an die fachwissenschaftliche Diskussion nicht zu verpassen, eine selbstbewusste Forschungskultur zu etablieren und hieraus auch den schulpraktischen Bezug nachhaltig sichern zu können.

Andererseits knüpfte Ingrid Hemmer an die Frage der Relation Fachdidaktik-Schule an, indem sie auf das Selbstverständnis und den Bildungsauftrag der Geographiedidaktik und damit auch wieder auf den Schulbezug hinwies. Sie stellte heraus, dass bereits in den 1980er Jahren von Helmuth Köck das Selbstverständnis der Geographiedidaktik als Auseinandersetzung mit geographischem Lernen und Lehren und den dazugehörigen Voraussetzungen etc. umschrieben

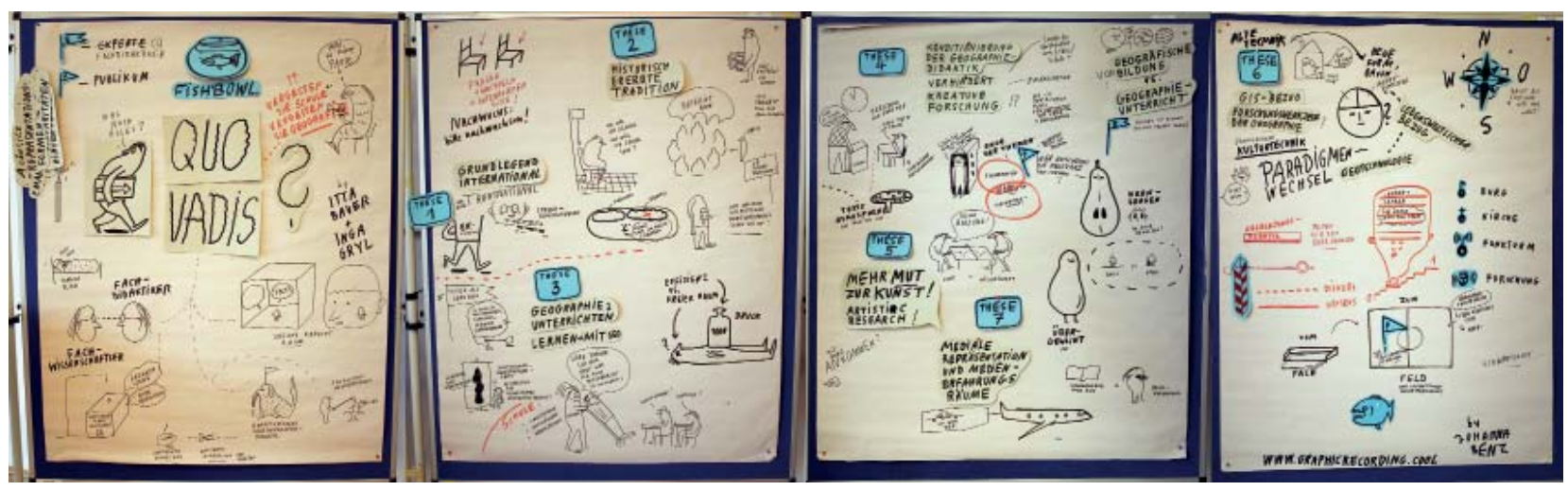

Abb. 5: Illustration der Gesamtdiskussion auf einer, Forschungslandkarte' (finales Graphic Recording); Zeichnung: Johanna Benz. 
wurde. Eine ,Konditionierung' durch den heutigen Unterricht wäre damals ebensowenig gegeben wie heute.

Ingrid Hemmer: Und wir sind in dem Spagat, insofern würde ich Ihnen Recht geben. Wenn wir jetzt einen Schulgeographen hier sitzen hätten - wahrscheinlich sind auch Kolleginnen und Kollegen aus der Schule da-die kommen manchmal mit dem Anspruch auf uns zu, "Ihr müsst jetzt für uns das und das tun, wir erwarten von euch das und das". Diese Erwartung haben wir aber nicht unkritisch hingenommen. (...) Stattdessen ist da sehr viel Diskussion, sehr viel Kooperation. Aber das beschränkt sich nicht auf den engeren Bereich des Unterrichts. Ich glaube, ich habe mich in meiner 30-jährigen Praxis in der Forschung noch nie eingeschränkt gefühlt (lacht), ganz im Gegenteil.

Den prinzipiellen Nutzen der Zusammenarbeit zwischen Fachwissenschaft und Fachdidaktik wiederum und die Problematik der Dichotimisierung zwischen beiden betonte eine Lehrerin ${ }^{6}$ aus dem Auditorium:

Lehrerin: Ich bin ihnen dankbar, dass Sie diese Diskussion wieder geöffnet haben, weil ich als Lehrkraft gedacht habe, dass wir doch alles Geographen sind, und wir ein Ziel haben, und das ist der Geographieunterricht. (...) Ich fände es einfach gut, wenn wir alle Hand in Hand arbeiten würden, auch wenn das etwas pathetisch klingt, aber Perspektiven, die aus der Fachwissenschaft in die Fachdidaktik geholt werden, sind doch genauso wichtig wie andersherum. Wir wollen zusammen etwas schaffen, wir wollen zusammen Schlüsselprobleme attackieren. Das können wir nur, wenn wir den Background haben, und den haben wir teilweise nicht mehr in dem Maße, wenn wir in der Didaktik arbeiten. Dann brauchen wir eben wieder die Fachwissenschaft, die Fachlichkeit, die uns vielleicht teilweise abhandengekommen ist. Deswegen würde ich an alle appellieren: Wir können zusammen forschen, wir können zusammen etwas schaffen. Schade nur, dass es immer diese Dichotomisierung gibt zwischen Fachwissenschaft und Fachdidaktik. (...)

Das Tagungsthema aufgreifend, brachte Ute Wardenga erneut die wissenschaftshistorische Perspektive ein, die Abgrenzungstendenzen der Geographiedidaktik zwar nicht legitimiert, aber ihre Existenz zumindest verständlich macht. Zugleich zeigte sie Alternativen auf.

\footnotetext{
6 Leider konnten wir den Namen der Lehrerin im Nachgang zur Fishbowl-Diskussion in Jena nicht ausfindig machen. Wir haben uns über die Wortmeldung der Lehrperson sehr gefreut und hoffen, dass dieser sprachlich leicht überarbeitete Diskussionsbeitrag auch in ihrem Sinne in die Veröffentlichung aufgenommen wurde.
}

Ute Wardenga: (...) Kann es sein, dass hier wieder Grenzen angesprochen werden? Das ist ja auch das schöne Tagungsthema. Gut, dann reden wir doch einmal über Grenzen. Ich habe das mit meinem Eingangsstatement ja auch in gewisser Weise provoziert. Aber denken wir doch mal zurück an die Phase, in der die Geographiedidaktik sich als eigenständiges Feld wissenschaftlicher Expertise herausgebildet und von der Fachwissenschaft emanzipiert hat. Das war die Phase der 1960er und 1970er Jahre, wo die Geographiedidaktik ein legitimer Gegenstand einer universitären Lehrer(innen) ausbildung wurde. Damit konnten Fachwissenschaftler (!) nicht mehr sagen: „Okay, wir bilden hier Lehrer aus und alles das, was für Didaktik notwendig ist, lernen die Studierenden dann im Referendariat. "Geographiedidaktik hat sich also zwischen Fachwissenschaft und Referendariat geschoben, als eine eigene Form von Expertise. Damit Eigenständigkeit entwickelt werden kann, müssen Grenzziehungen erfolgen. Sie konstituieren das Eigene im Spiegel des durch die Grenzziehung ausgeschlossenen Anderen. Was ich jetzt erkenne, ist, dass man zunehmend zwischen Fachwissenschaft einerseits und Geographiedidaktik andererseits wieder ins Gespräch kommen könnte. Und das, was ich meine beobachtet zu haben, ist eine Art von Öffnung. (...) Ich mache nun einmal eine steile These auf. Die Geographiedidaktik könnte sich aus einer Art abgeschlossenem Teilbereich innerhalb eines Fachs zu etwas entwickeln, das wir auch anderswo sehen: nämlich zu einem Feld der Expertise mit unscharfen Grenzen, mit Überschneidungsbereichen und mit einem dadurch plötzlich aufpoppenden, neuen multiperspektivischen Kern. Ich provoziere jetzt einmal: Offensichtlich gehen die Zeiten, in denen Geographiedidaktik als eigenständiger, territorial homogenisierter ,Nationalstaatscontainer mit festen, quasi linienhaft gezogenen Grenzen imaginiert werden konnte, zu Ende.

Die Debatte um die Positionierung der Geographiedidaktik in Abgrenzung von den Fachwissenschaften zog sich trotz dieser sehr auf Kooperation, Austausch und Wandel angelegten Statements im Folgenden weiter durch die Diskussionsbeiträge, was hier nur verkürzt wiedergegeben werden kann. Ingrid Hemmer brachte hier als neuen Punkt die politische Perspektive der Problematik ein.

Ingrid Hemmer: Die Zahl der Geographiedidaktikprofessuren hat sich in den letzten Jahrzehnten von 80 auf 40 verringert. Es ist wichtig, den eigenen Standort nicht aufzugeben und nicht zu verlieren und deutlich zu sagen: „Das ist meine Expertise, das ist eure Expertise, wir können gerne darüber reden, aber nicht, dass der Grenzgang soweit geht, dass unser Profil völlig verschwindet. "Das ist gefährlich, weil wir ja auch für den Erhalt von Professuren und Qualifizierungsstellen in der Geographiedidaktik kämpfen müssen. 
Ingrid Hemmer erweiterte in der Folge die Argumentation der Relation Geographiedidaktik und Schule, indem sie die janusköpfige Stellung der Geographiedidaktik in Bezug auf Schule thematisierte. Diese biete nicht nur für Geographiedidaktiker/innen einen Raum für Grundlagenforschung und angewandte Forschung, sondern Geographiedidaktik und Geographieunterricht seien aus historisch gewachsenen Strukturen auch in einem wechselseitigen Abhängigkeitsverhältnis miteinander verbunden. Das äußere sich u.a. im gemeinsamen Ausbildungs- und Bildungsauftrag für die Schule (Unterricht) und die Universität (Forschung). Sie erinnert damit an eine Verantwortung, die Geographiediaktiker*innen tragen:

Ingrid Hemmer: Und da genau, da bewegen wir uns und das ist ein starkes Feld der Verantwortung, wenn wir zum Beispiel mitarbeiten, auch um Curricula und Lehrpläne zu entwickeln.

Thomas Jekel wies diesen Gedanken, auch als Referenz auf Verena Schreibers These, an anderer Stelle noch einmal pointiert aus:

Thomas Jekel: So lange wir in der Fachdidaktik sind, haben wir schon am Ende, die Notwendigkeit zu denken, dass es einen gewissen Endzweck gibt, und der ist Bildung, nicht wahr?

Im weiteren Verlauf wurden damit im Zusammenhang stehende Begrifflichkeiten und Grundsatzfragen z. B. zum Bildungsgehalt und -auftrag der Geographie sowie deren Legitimation noch einmal hinterfragt und kommentiert. Hierbei ging es Uwe Schulze im Hin- blick auf Bildung um die Frage der ,Verwertbarkeit und Verwertung/Instrumentalisierung von Bildungsleistungen, was auch auf die Kritik an der Kompetenzorientierung hinweist. Er führte diesen Zusammenhang am Beispiel von GIS ein.

Uwe Schulze: GIS sind einerseits Forschungswerkzeuge für die Fachwissenschaft Geographie (...). Andererseits wird GIS auch mit Medienkompetenz verbunden. Und so forscht man mit GIS auch im Bereich der Medienkompetenz und darüber hinaus (...) auch über räumliches Denken beispielsweise und im Bereich der Problemlösekompetenz. (...) Warum kann man daher nicht einfach sagen, es handelt sich beim Umgang mit Geoinformation um eine grundlegende Kulturtechnik, wobei die Technik-und Medienkompetenz natürlich eine Rolle spielen kann, aber es zudem auch um die mediale Artikulation von Selbst- und Weltverhältnissen, unter anderem geographischer Art, geht. Damit können wir eine Tür zur strukturellen Medienbildung aufmachen und nach dem Mehrwert der Nutzung und Aneignung von Geoinformationstechnologien fragen. Was ist eigentlich der Kern der Beschäftigung mit dieser Technologie? (...) Es ist nicht in erster Linie die technische Beherrschung dieser Instrumente und der Technologie, sondern eben auch all das, was da unten steht (Blick zur Wand), (...) Begegnung mit den räumlichen Informationen (...), eine kreative geographische Bildung, die auch neue Horizonte zu öffnen vermag. Deswegen habe ich auch bewusst das recht provozierende Wort des Paradigmenwechsels in meiner These verwendet.

An diesem Punkt der Diskussion überlagerten sich die Argumente von Uwe Schulze und Verena Schreiber,

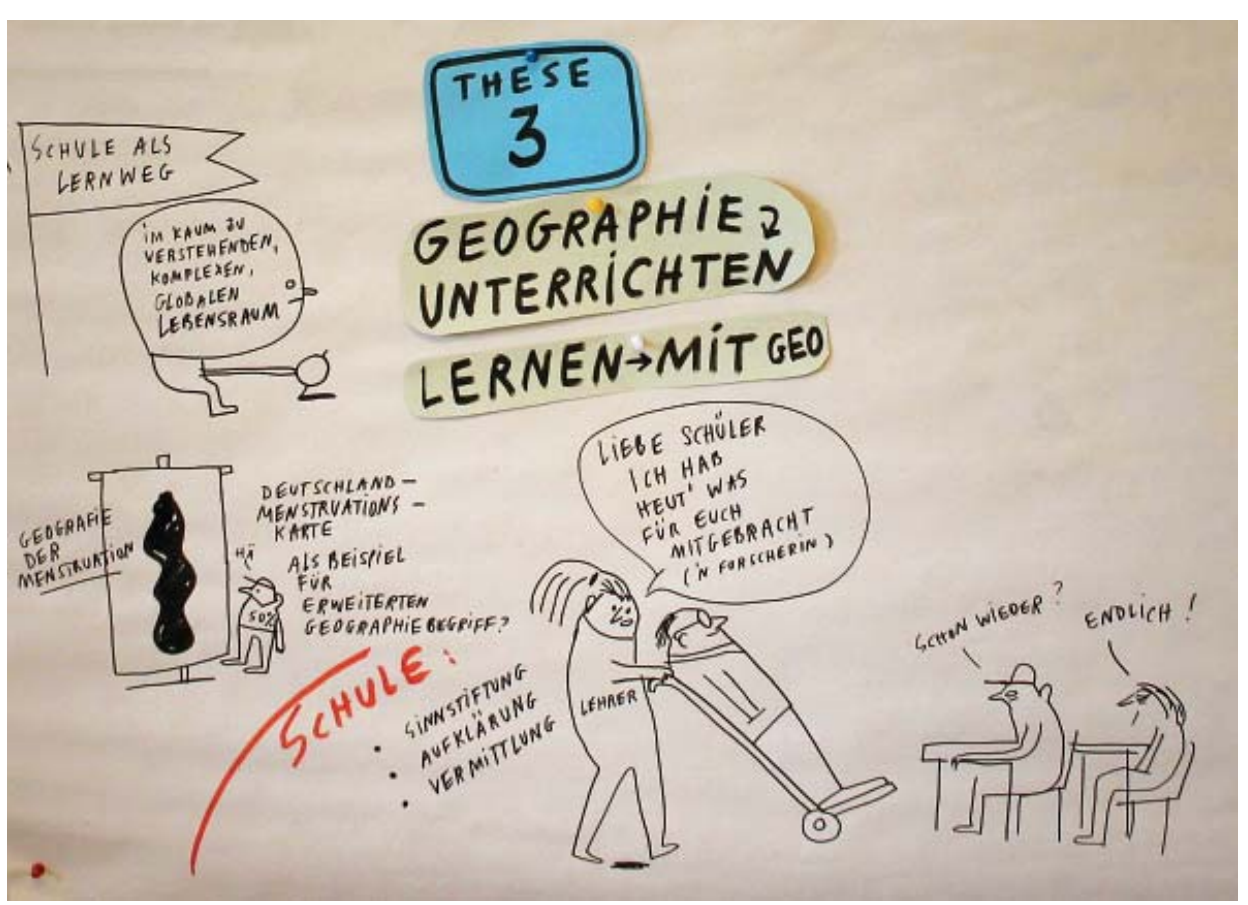

Abb. 6: Illustration der These von Thomas Jekel und der zugehörigen Diskussion; Zeichnung: Johanna Benz. 
die beide dafür plädierten, das Verhältnis von Geographie, Geographiedidaktik und Schule, kritisch zu hinterfragen. Verena Schreiber brachte die Problematik der Themensetzung ein.

Verena Schreiber: Wer entscheidet denn, was die relevanten Themen sind? Ich finde, dass diese Enge der Themen, dass Themen generell durch die Institution Schule schon stark vorgegeben sind. Das müsste man allerdings viel differenzierter betrachten: Wie genau kommen diese Themen zustande?

Damit wird der Blick auch auf die Frage gelenkt, ob der Geographiedidaktik nicht auch die Rolle zukommt, aktuelle Strömungen in der heterogen ausgefächerten bzw. intern multidisziplinär gewordenen Fachwissenschaft Geographie kritisch auf ihren Bildungsgehalt hin zu durchleuchten. Einige Geographiedidaktiker/ innen warfen in diesem Zusammenhang mehrfach die Frage des spezifisch ,Geographischen' an geographischer Bildung auf:

Uwe Schulze: Was ist also geographische Bildung? (...)

Thomas Jekel: Warum geographische Bildung? Sag mir, warum Bildung in dem Fall geographisch ist. Ich möchte es einfach einmal verstehen!

Thomas Jekel: Bildung gefällt mir. Aber warum setzen wir automatisch immer, geographisch' als Adjektiv davor? Warum muss das sein?

Abschließend konnte diese Frage nicht beantwort werden und ihre Beantwortung war auch nicht das Ziel der Diskussionsrunde. Gleichwohl sind in der Diskussion verschiedene Argumente im Hinblick auf Bildung vorgebracht worden, die wir hier auf Wunsch der Autor/innen nicht anführen dürfen bzw. dadurch nicht näher ausführen können. Die ,Leerstellen' des kooperativen Schreibens und Nachbearbeitens einer öffentlich geführten Diskussion treten an dieser Stelle sichtbar zu Tage. Die Relevanz, auch den Bildungsbegriff in der Kopplung der ,geographischen Bildung näher zu betrachten, zu hinterfragen und zu spezifizieren, statt allein den Fokus auf,geographisch' zu legen, wurde dabei jedoch sehr deutlich.

Abschließend kristallisierte sich als weitere Grundsatzfrage die Freiheit von Forschung (und Lehre) in der Geographiedidaktik heraus.

Leif Mönter: Ich würde gerne aufgreifen, wie sehr Forschung über strukturelle Bedingungen abgesteckt ist. Es klingt immer, als ob hier ein ganz herrschaftsfreier Diskurs stattfinden kann und unter optimalen Bedingungen Forschung betrieben werden würde. Aber jedel jeder von uns weiß, dass dem nicht so ist. Unter welchen, auch Karrieregesichtspunkten, findet Forschung statt, wie werden Moden eingebunden, (...) wofür kann ich Drittmittel aquirieren, welche Frage, welches Thema ist überhaupt gefragt? Unsere Diskussion kann nicht so stehen bleiben, als seien wir quasi in einem Reich der Freiheit, denn das blendet die politökonomische und soziokulturelle Einbettung von Wissenschaft aus.

\section{Raum und Zeit für ein Fazit}

Gegen Ende der Diskussion zeigte sich eine neue Ebene, die hilfreich war, um einerseits Take-Home-Messages aus der Diskussion abzuleiten und andererseits auch erste Rückmeldungen auf die Debatten in der Fishbowl-Runde und die Methode selbst zu erhalten. Detlef Kanwischer beispielsweise griff sowohl die Metapher der ,Forschungslandkarte` als auch die der Grenzen auf:

Detlef Kanwischer: Vielleicht eine Anmerkung, ein kleines Resümee (...) und das möchte ich den vielen jungen Leuten hier mitgeben: Macht das, was euch interessiert und nicht, was da an der, Forschungslandkarte'steht. (...) Und wenn es ganz konkret um eine ,Forschungslandkarte' geht, dann würde ich auch (...) über die Grenzen hinaus auflisten, was dort alles möglich ist. Wenn wir in unserer Disziplin gut sein wollen, dann müssen wir Grenzen überschreiten, dann müssen wir Ideen aus anderen Fächern adaptieren und deswegen ist unsere, Forschungslandkarte' hier viel zu statisch, deswegen muss man auch über die Grenzen hinausgehen und aufzeigen, was darüber hinaus alles möglich wäre. $\mathrm{Ob}$ das alles sinnvoll ist oder nicht, das muss dann jedel jeder Einzelne für sich herausfinden.

Inga Gryl: Ich denke, das ist ein gutes Stichwort, das du uns nun gegeben hast: Wir müssen de facto kritische Kartographie auf unsere Karte anwenden, also schauen, was hinter den Grenzen der Karte ist. Aber wir verstehen diese, Landkarte' natürlich nicht als Wegweiser. Wir sind uns dessen bewusst, dass eine und diese "Landkarte" immer nur einen Ausschnitt darstellt, dass sie eine bestimmte Perspektive darstellt, dass sie kein Abbild ist und auch kein Abbild der Forschungslandschaft. Sie ist ein Schnappschuss dieser Diskussionsrunde und soll zu weiteren Diskussionen einladen und anregen.

Eva Nöthen: So versteht sich die heutige Veranstaltung, in meinen Augen doch auch als eine Antwort, auf das, was in Berlin passiert ist. Der Rahmen der Diskussion war damals deutlich enger gefasst und es ging eher darum, das Feld geographiedidaktischer Forschung abzustecken und einzugrenzen. Ich denke, die Diskutan- 
ten/Diskutantinnen für die heutige Fishbowl sind ausgewählt worden, um dieses Feld wieder zu öffnen und so zu zeigen, wo wir Didaktikerlinnen uns neue Horizonte erschließen können, ohne dabei ,Forschungsterritorien" gegeneinander zu verteidigen.

Obgleich wir, entsprechend dem Fazit von Eva Nöthen, versucht haben, in Kohärenz mit der Rahmung der Tagung und unserer eigenen Auffassung von Forschung und Geographiedidaktik als Disziplin bestehende Grenzen hinter uns zu lassen, wurden auch uns Grenzen aufgezeigt. Wenig erfolgreich waren wir etwa in unserem Bestreben, Vertretern/Vertreterinnen des Nachwuchses mehr Gehör zu verschaffen. Darauf wies Stephan Schurig in der offenen Feedback-Runde zur Fishbowl-Diskussion ausdrücklich hin.

Stephan Schurig: Ich weiß nicht, wie ihr damit umgeht, aber die Idee, zu forschen, was ich will, hat auch viel mit sozialen Privilegien zu tun. Wenn man so auf die Struktur schaut, wer hier sitzt, und wer sich dazugesetzt hat, dann ist das hauptsächlich die eingesessene Fachdidaktik, dann sind das Leute, die schon lange forschen. Das ist eigentlich sehr schade, finde ich, dass sich junge Leute eher wenig beteiligt haben, um eben auch diese Machtstruktur zu durchbrechen. Ich weiß nicht, wie man es hätte lösen können, mehr Zeit, mehr Stühle oder weniger Referenten, ich weiß es nicht. Es ist eine Beobachtung, über die man einmal nachdenken könnte.

Ein weiterer Nachwuchsvertreter, Lukas Recknagel, suchte im Anschluss an die Diskussionsrunde das Gespräch mit uns. Er wies darauf hin, dass seiner Ansicht nach nicht nur der Austausch zwischen den Generationen zu kurz kam, sondern auch durch die abstrakte Diskursführung eine große Hemmschwelle für jüngere Geographiedidaktiker/innen aufgebaut wurde. Seine Eindrücke, Überlegungen und Anregungen fasste er im Nachgang für uns schriftlich zusammen:

Lukas Recknagel: Ich empfand die Hemmschwelle, sich an der Diskussion zu beteiligen, als sehr hoch. Das zeigte sich auch darin, dass sich nur sehr wenige ,von uns Jungen' eingebracht haben. Das lag vor allem daran, auf welchem Level die Debatte die meiste Zeit geführt wurde, nämlich einer Metaebene, der allein zu folgen sehr schwer war, ganz abgesehen davon, sich darin zu verorten und mit eigenen Impulsen einzubringen. Darüber hinaus stellt sich grundsätzlich die Frage, ob bei einer solchen zukunftsgerichteten Leitfrage die Stimme des Nachwuchses nicht einen prominenteren Platz gebraucht hätte, denn auch unsere Ideen, Interessen und Sorgen werden ja früher oder später eine entscheidende Rolle spielen.
Ein Gedanke noch zum Rahmenthema, Grenzen': Ich habe häufig den Eindruck, dass von Nachwuchsseite (auch jenseits wissenschaftlicher Kreise) erwartet wird, viele neue Ideen einzubringen und so gewissermaßen die Grenzen zu überschreiten. Das ist legitim, denn das Ausloten von Grenzen ist ja auch Teil eines, jungen oder jugendlichen' Selbstverständnisses. Jetzt habe ich jedoch auch den begründeten Verdacht, dass gerade das Überschreiten von Grenzen und das Ausprobieren neuer Wege im wissenschaftlichen Feld nur bedingt auf Begeisterung stoßen. Jenseits vom Konsens, dass eine Forschungslücke bearbeitet werden sollte, werden immer wieder gerade die unkonventionellen Ansätze sehr kritisch beäugt und viele Projekte nähern sich so schrittweise wieder den bewährten Methoden und Designs. Ich kann letztlich aber nicht beurteilen, ob dieser Umstand bedeutet, dass wir als Forscherinnen und Forscher einfach unvorbereitet auf Neues sind oder aber die bewährten Ansätze so unumstößlich, dass andere Ansätze grundsätzlich ausgeschlossen werden. Unabhängig davon ist das Überschreiten von Grenzen gerade ohne Erfahrung vielleicht auch einfach ein zu hoher Anspruch.

Wir sind sehr dankbar für diese Rückmeldungen des wissenschaftlichen Nachwuchses und halten es für essentiell, diesem in einem zukünftigen Format, dieser Argumentation folgend, mehr Raum zu geben. Dies sollte unserer Ansicht nach aber nicht nur in expliziten Nachwuchsveranstaltungen geschehen, sondern auch in größeren Tagungen als generationenübergreifendes Projekt ins Programm aufgenommen werden, um die Diskussion auf eine ,multi-sensible 'Art und Weise konstruktiv weiterzuführen.

Generell möchten wir dies zum Anlass nehmen, die Anlage der Diskussion abschließend zu reflektieren: Unsere Rolle als Moderatoren/Moderatorinnen haben wir stets als möglichst vermittelnd, möglichst neutral, aber auch möglichst die Diskussion voranbringend wahrzunehmen versucht, wobei einige dieser Funktionen bereits naturgemäß als Balanceakt zu verstehen sind. Schon bei der Vorbereitung galt es, Entscheidungen zu treffen, etwa dahingehend, nicht alle Personen, die etwas zum Thema beitragen könnten - und das sind sehr viele - und gegebenenfalls auch würden, einladen zu können. Unsere Planung bestand in der Vermeidung einer Reproduktion der Berliner Diskussion - denn es sollte ja um eine weitere Perspektive und einen Mehrwert für alle Beteiligten und Zuhörenden durch Weiterentwicklung gehen - aber auch in Vermeidung der Konstruktion eines bloßen Gegenmodells. Im Sinne einer eingangs erläuterten ,multisensiblen Geographiedidaktik' haben wir versucht, eine offene Atmosphäre zu schaffen, die sich Zeit nehmen kann für die Entwicklung vielfältiger Perspektiven, sind uns aber bewusst, dass zeitliche Grenzen den Austausch limitierten. 
Für die Zukunft würden wir uns wünschen, dass der gedankliche Austausch unter Geographiedidaktikern/Geographiedidaktikerinnen verschiedener Alters- und Karrierestufen konstruktiv fortgesetzt wird. Auch wenn es in der Geographiedidaktik gilt, sich manchmal mit ,unbequemen Grenzgängen' zwischen Fachdidaktik, Fachwissenschaft und der Praxis von Geographie und Unterricht auseinanderzusetzen, so bleibt es doch ein spannendes Unterfangen, die eigenen Grenzen immer wieder neu auszuloten, um sich als scientific community gemeinsam vor Augen zu führen, wie die eigene ,Forschungslandkarte' ständig in Bewegung und offen für Dekonstruktion ist. Wir würden uns gerne für eine Geographiedidaktik aussprechen und einsetzen, die hierbei grundsätzlich offen für, aber auch kritisch-reflexiv gegenüber neuen Anregungen aus den verschiedensten Bereichen von Forschung, Politik und Gesellschaft ist. Eine so verstandene Geographiedidaktik nimmt nicht nur neue Impulse aus anderen Bereichen auf, sondern wirkt auch inspirierend über die eigenen Grenzen hinaus.

Vor diesem Hintergrund möchten wir alle Teilnehmenden der Fishbowl-Diskussion und alle weiteren Interessierten dazu aufrufen, eigene Beiträge zur Thematik oder zum Format (z. B. zum Stand und zu den Zukunftsperspektiven der Geographiedidaktik, explizite Antworten auf Diskussionsbeiträge) beispielsweise an dieser Stelle einzureichen, und die Diskussion auf diese Weise weiterzuführen.

\section{$7 \quad$ Literatur}

Bagoly-Simó, P. \& I. Hemmer (2016): Geographiedidaktische Forschung - quo vadis? Ergebnisse einer Expertendiskussion. In: Zeitschrift für Geographiedidaktik| Journal of Geography Education (ZGD), 44/3, 55-64.

Browne, K., N. Banerjeab, N. McGlynn, B. Sumita, L. Bakshi, R. Banerjee \& R. Biswas (2017): Towards transnational feminist queer methodologies. In: Gender, Place \& Culture 24/10, 1376-1397.
Budke, A. \& M. Kuckuck (Hrsg.) (2017): Sprache im Geographieunterricht. Bilinguale und sprachsensible Materialien und Methoden. Münster: Waxmann.

Budke, A. \& G. Weiss (2014). Sprachsensibler Geographieunterricht. In: Michalak, M. (Hrsg.), Sprache als Lernmedium in allen Fächern. Baltmannsweiler: SchneiderVerlag Hohengehren, 113-133.

Cahill, C. (2004): Defying gravity? Raising consciousness through collective research. In: Children`s Geographies 2/2, 273-286.

Dickel, M., L. Keßler, F. Pettig \& F. Reinhardt (Hrsg., 2018): Grenzen markieren und überschreiten - Positionsbestimmungen im weiten Feld der Geographiedidaktik. Tagungsbeiträge zum HGD-Symposium 2017 in Jena. Münster: readpress. (In Druck)

Hodel, J. \& P. Haber (2007): Das kollaborative Schreiben von Geschichte als Lernprozess. Eigenheiten und Potenzial von Wiki-Systemen und Wikipedia. In: Merkt, M., K. Mayrberger, R. Schulmeister, A. Sommer \& I. van den Berk (Hrsg.): Studieren neu erfinden - Hochschule neu denken. Münster u. a.: Waxmann, 43-53.

Lowry, P. B., A. Curtis \& M. R. Lowry (2004): Building a Taxonomy and Nomenclature of Collaborative Writing to Improve Interdisciplinary Research and Practice. In: Journal of Business Communication 41/1, 66-99.

Lohse, T. \& C. von Buchholz (2007): Kollaboratives Schreiben an wissenschaftlichen Texten. „Neue Medien“" und „Neue Lehre“ im Fach Geschichte. In: Merkt, M., K. Mayrberger, R. Schulmeister, A. Sommer \& I. van den Berk (Hrsg.): Studieren neu erfinden - Hochschule neu denken. Münster u. a.: Waxmann 2007, 76-84.

Schröder, B. (2016): Machtsensible geographiedidaktische Konzepte des interkulturellen Lernens - Potenziale einer postkolonialen Perspektive. In: GW-Unterricht 144/4, 15-28.

Townsend, J. mit U. Arrevillaga, J. Bain, S. Cancino, S. F. Frenk, S. Pacheco \& E. Percz (1995): Women`s voices from the rainforest. London: Routledge.

\section{Danksagung}

Der VGD-CH unterstützte diesen Beitrag mit einem Druckkostenzuschuss. 